\title{
Capillary condensation experiment for inverse modelling of porous building materials
}

\author{
Hauke Hirsch ${ }^{1 *}$, Rüdiger Heyn ${ }^{1}$ and Paul Klõšeiko ${ }^{2}$ \\ ${ }^{1}$ Dresden University of Technology, Institute of Building Climatology, 01060 Dresden, Germany \\ ${ }^{2}$ Tallinn University of Technology, Department of Civil Engineering and Architecture, 19086 Tallinn, Estonia
}

\begin{abstract}
Hygrothermal simulations are of major importance for critical problems in building physics, such as the application of internal insulation in heritage buildings. These simulations require numerous material parameters that are challenging to determine. We present measurements of typical internal insulation materials, calcium-silicate and autoclaved aerated concrete, which we expose to a warm, humid climate on one side and a cold temperature on the other side. We measure the moisture gain over time and determine the moisture profile at experiment end. In an inverse modelling approach, the measurements are used to identify material parameters, in particular vapour conductivity and capillary conductivity as a function of moisture content. We found the measurements of crucial importance for the accurate determination of these parameters. When the parameters rely only on isothermal measurements such as the drying experiment, the model fails to predict the capillary condensation process. We demonstrate this on a dataset from another study with interior insulation subjected to changing boundary conditions. The model calibrated with capillary condensation data reliably reconstructs measurements while the drying-calibrated model drastically underestimates the moisture content.
\end{abstract}

\section{Introduction}

Interior insulation materials are gaining importance with the requirement of retrofitting heritage buildings. Their application in practice, however, is still a sensitive topic, as wrong dimensioning may lead to serious moisture damage and mould problems of the construction. In order to avoid these problems, capillary active materials, such as calcium-silicate are used, which feature a high capillary conductivity even in low moisture content ranges. For their dimensioning, it is recommended to rely on hygrothermal simulations [1]. The predictions of such simulations strongly depend on the parameters, which needs to be determined for each single material using laboratory experiments [2]. As shown by Klõšeiko et al. [3], [4] in some cases simulations drastically fail to predict the measured moisture content under realistic boundary conditions.

Common experiments for material parameter determination are the vapour cup test [5], water absorption test [6] and drying test [7]. In order to find the correct parameters, it is attempted to reconstruct their results with the model. However, these experiments are mostly isothermal with vapour and liquid fluxes occurring in the same direction, which hardly allows distinguishing between both. Hence, as it has been shown by Binder et al. [8] [9] as well as by Klõšeiko et al. [4], the determined parameter may not be valid for modelling a non-isothermal experiment and hence the hygrothermal simulation could not reconstruct real-world measurements.

Therefore, a non-isothermal experiment was introduced [9] [10], also called capillary condensation redistribution test (CCR). Material samples are on one side subjected to a warm, humid climate, while at the other side a constant cold temperature is applied. This is close to the practical application of these materials, where vapour and liquid fluxes occurring in opposite directions.

We set up a CCR test for three different materials, which are two types of calcium-silicate (CaSi-A, CaSi-B) and autoclaved aerated concrete (AAC). In contrast to [8], we used samples with larger dimensions to reach closely one-dimensional behaviour. Moreover, we apply an inverse modelling approach using an optimization algorithm for parameter identification. Within the algorithm, the vapour cup, drying, and CCR experiments are modelled with DELPHIN 6 [11] [12] and a material parameter set is identified that matches all measurement results with sufficient accuracy.

The article describes the experimental setup, the measurement procedure and the obtained results in detail. Next, the functional approach for the vapour and liquid conductivity as well as the optimization technique is introduced. Finally, we present the parameters determined by inverse modelling and compare the simulation results against our measurements.

\section{Experiment}

\subsection{Preparation of samples}

We measured three different materials:

- CaSi-A: calcium-silicate, not commercially available but measured by various laboratories in [13]

- CaSi-B: calcium-silicate, commercially available

- AAC: autoclaved aerated concrete, commercially available

The basic material properties are shown in Table 1. The thermal conductivity $\lambda$ is given at dry state. The vapourresistance factor $\mu$ was measured at boundary conditions

\footnotetext{
* Corresponding author: hauke.hirsch@tu-dresden.de
} 
of 0 and $35 \%$ RH. The moisture content at effective saturation $\theta_{\text {eff }}$ was determined through underwater submersion. We cut the materials into samples of size $100 \mathrm{~mm} \mathrm{x}$ $100 \mathrm{~mm} \times 50 \mathrm{~mm}$. At all lateral sides except for the surface, we painted the samples using an emulsion paint. Next, we coated the sides with paraffin and laminated them using a flexible EPDM isolation material, which is vapour- and watertight and has a thermal conductivity of around $0.03 \mathrm{~W} /(\mathrm{m} \mathrm{K})$. Finally, the samples are glued on the sample carrier, a $100 \mathrm{~mm} \times 100 \mathrm{~mm}$ aluminium plate with a bore for temperature sensor placement. The finished sample is shown in Figure 1.

Table 1. Thermal conductivity $\left(\lambda_{\text {dry }}\right)$, density $(\rho)$, vapourresistance factor $(\mu)$ and effective saturation $\left(\theta_{\text {eff }}\right)$ of the measured materials.

\begin{tabular}{lcccc} 
& $\begin{array}{c}\boldsymbol{\lambda}_{\text {dry }} \\
\mathrm{W} /(\mathrm{m} \mathrm{K})\end{array}$ & $\begin{array}{c}\boldsymbol{\rho} \\
\mathrm{kg} / \mathrm{m}^{3}\end{array}$ & $\begin{array}{c}\boldsymbol{\mu}_{\mathbf{0} / 35 \% R H} \\
-\end{array}$ & $\begin{array}{c}\boldsymbol{\theta} \text { eff } \\
\mathrm{m}^{3} / \mathrm{m}^{3}\end{array}$ \\
\hline CaSi-A & 0.069 & 270 & 2.2 & 0.90 \\
\hline CaSi-B & 0.059 & 189 & 2.4 & 0.93 \\
\hline $\mathbf{A A C}$ & 0.045 & 104 & 2.9 & 0.25 \\
\hline
\end{tabular}

\subsection{Experimental setup}

Experimental boundary conditions are a warm, humid climate on the upper side and a cold temperature at the bottom side. Therefore, we conducted the experiment inside a climate chamber using a cooling plate made of aluminium with a size of $390 \mathrm{~mm} \times 450 \mathrm{~mm}$. The setup with the samples is shown in Figure 2. The cooling plate has a meander-type channel, which is filled with a waterglycol mixture and connected to a laboratory chiller. This allows us to accurately determine the surface temperature with a spatial uniformity of $<0.2 \mathrm{~K}$. The material samples are glued to a sample carrier made of aluminium. The two triangular shaped edges of this carrier form a dovetail connection with counterparts that are screw fastened to the cooling plate. If the screws are slightly loose, the counterparts can be slid along slot holes. With this arrangement it is possible to quickly unlock the sample carriers from the cooling plate and thus to achieve only short interruptions for weighting the samples. At the same time, a secure and reproducible thermal contact between sample carrier and cooling plate is achieved.

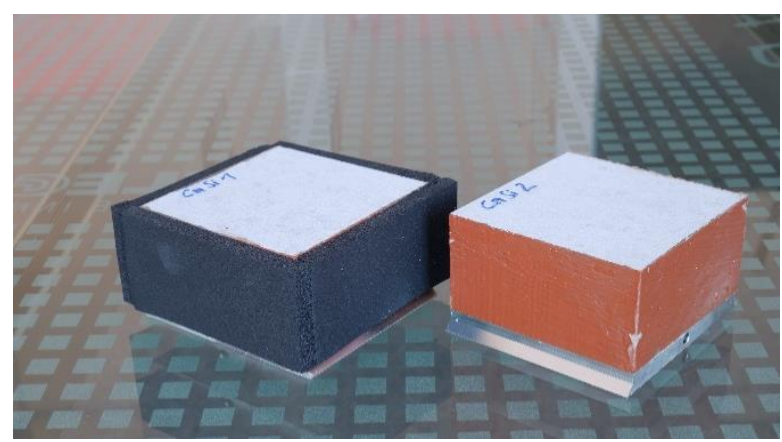

Fig. 1. Sample of calcium-silicate (CaSi-B) on the sample carrier, before (right) and after (left) isolating it with EPDM.

Between the samples of different material and at the lateral sides, the samples are separated by an XPS-isolation with a thermal conductivity of $0.035 \mathrm{~W} /(\mathrm{m} \mathrm{K})$. Finally, the whole setup is kept tight by fixing the lateral sides with plastic panels.

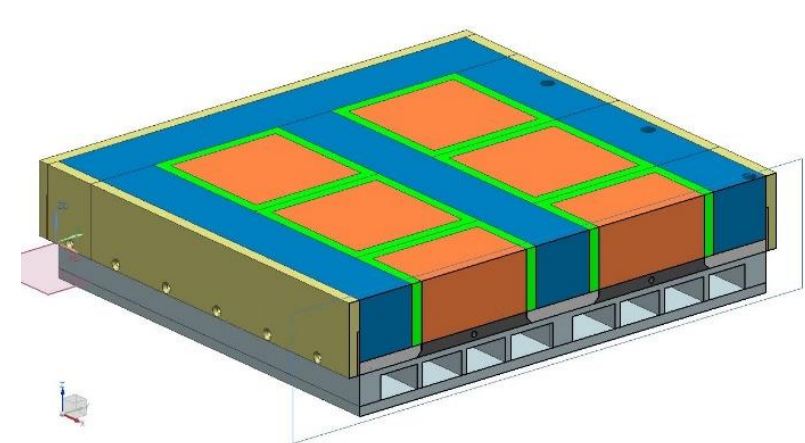

Fig. 2. Vertical cut through the experimental setup on the cooling plate. The samples (orange) are isolated each with an EPDM layer (green) and surrounded by XPS-isolation (blue). Sample carriers (black) and counterparts (grey) form a dovetail connection. The setup is surrounded by plastic panels (yellow).

Figure 3 depicts the final setup inside the climate chamber. We placed fans on top of the setup, in order to raise the air velocity at the sample surface, so that the mass and heat transfer resistances are reduced. We recorded the bottom temperature of four samples at the edges of the setup. Therefore, cylindrical-shaped sensors were plugged into the borehole in the sample carrier. The air temperature and relative humidity were measured above the sample surface. For temperature measurement, we used Pt100-Sensors, which were calibrated before the start of the measurements. Relative humidity was measured using a capacitive relative humidity sensor. All measurement values were continuously recorded.

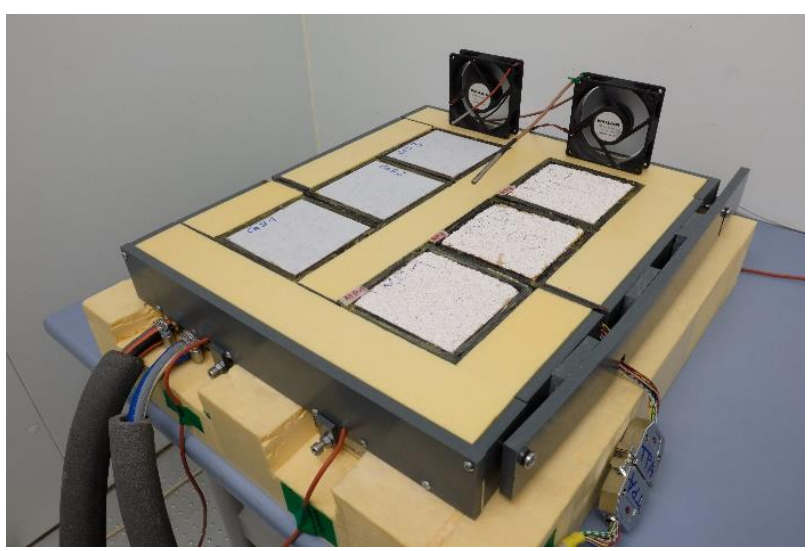

Fig. 3. Final experimental setup in the climate chamber.

\subsection{Measurement procedure}

The measurement was conducted over a period of 72 days. With an interval of between one and three days, we took the samples off the cooling plate, removed condensed water from the sample carriers and determined their weight using a scale with $0.001 \mathrm{~g}$ resolution. We set the boundary conditions to $22{ }^{\circ} \mathrm{C}$ and $70 \%$ relative humidity inside the climate chamber and $5{ }^{\circ} \mathrm{C}$ on top of the cooling plate. Temperatures stayed in a range of $\pm 0.1^{\circ} \mathrm{C}$ and the relative humidity in a range $\pm 2 \%$ over the entire 
measurement period except during the time when we entered the climate chamber to weigh the samples, which took about 20 min respectively.

After finishing the experiment, the samples were immediately cut into five horizontal slices of about $10 \mathrm{~mm}$ each. We measured their mass before and after drying them at $45{ }^{\circ} \mathrm{C}$. This allowed us to determine the spatial water content profile along the sample height.

\subsection{Measurement results}

Figure 4 and Figure 5 show the measured volumetric moisture content and its time derivative for all materials. During the first 15 days, the materials behave largely similar. Due to the vapour pressure gradient, condensation occurs in the sample and the moisture content increases. The time derivative of the moisture content, however, shows a clear decrease, which is due to a rising relative humidity in the material that causes the vapour pressure gradient to decrease with time.

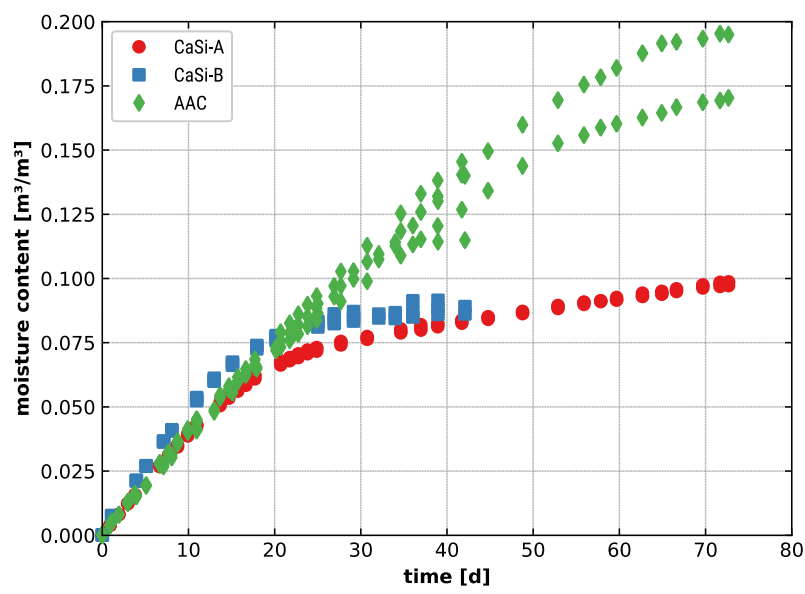

Fig. 4. Average volumetric moisture content of the materials over the measurement period.

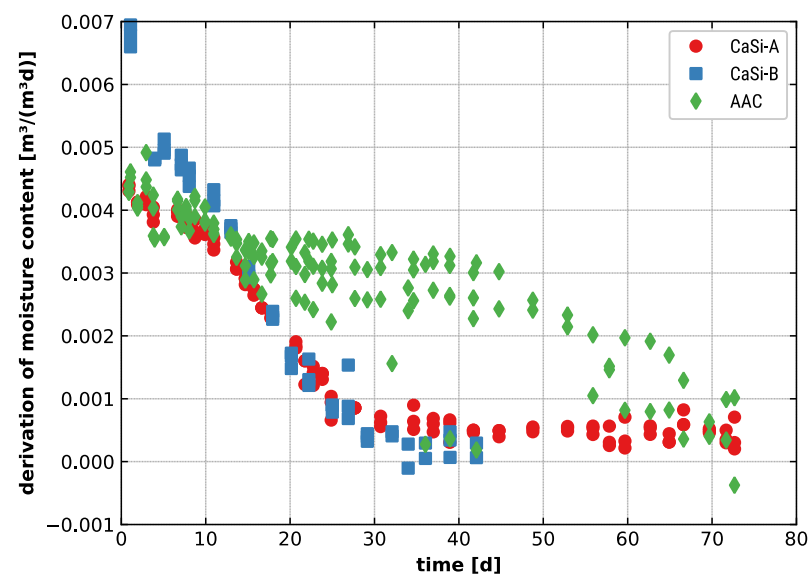

Fig. 5. Time derivative of the volumetric moisture content in $\mathrm{m}^{3} /\left(\mathrm{m}^{3 *} \mathrm{~d}\right)$ over the measuring period.

After 15 days, the materials clearly differ from each other. The moisture gain of CaSi continues to decrease until it asymptotically approaches zero. Hence, the average moisture content of CaSi stays at a significantly lower level than that of AAC. The further decrease of moisture gain in $\mathrm{CaSi}$ can be explained by both a decreasing vapour pressure gradient and an increasing capillary conductivity. The samples of AAC noticeably differ, in particular at higher moisture contents. The moisture gain stays at a constant level before it continues to decrease after day 40 . We assume this behaviour to be caused by multiple reasons: a limited capillary conductivity and a large number of pores of similar size, i.e. a step in the moisture storage function, which maintains the vapour pressure gradient constant for a limited period. It should also be noted that the AAC used here has a lower thermal conductivity, which causes lower temperatures inside the material and hence a higher vapour pressure gradient.

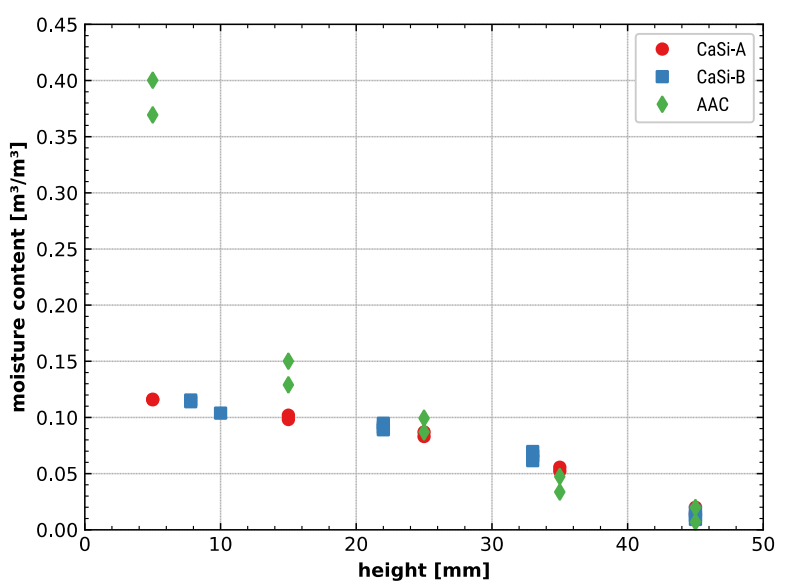

Fig. 6. Spatial profile of volumetric moisture content over sample height at the end of the measurement.

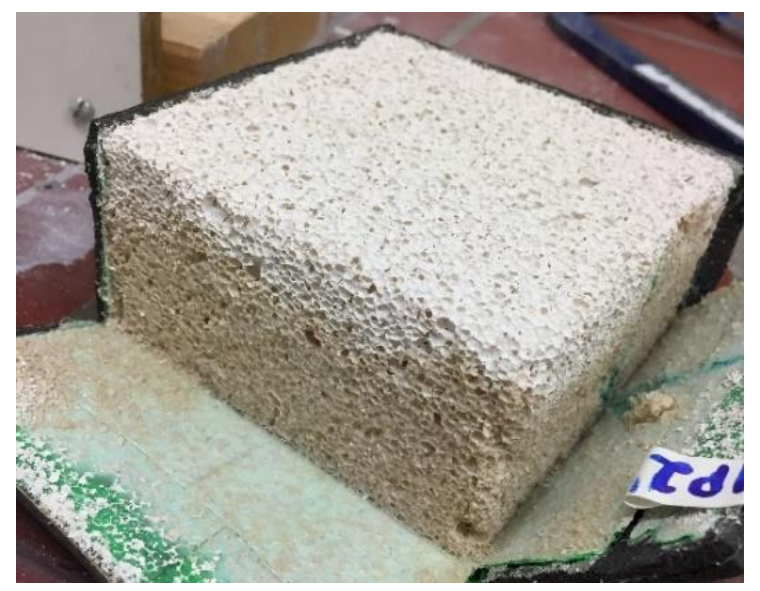

Fig. 7. Sample of AAC at the end of the measurement, moist side can clearly be distinguished.

Figure 6 shows the profile of the water content over sample height at the end of the measurement. The difference in moisture content between the materials is obvious. The moisture inside the AAC appears to stay predominantly at the bottom, which confirms the assumption of the material's limited capillary conductivity. The moisture distribution can be seen even when looking at the sample at measurement end as shown in Figure 7. It should be noted that the moisture content achieved here is higher than the one obtained through underwater submersion (Table 1). 


\section{Inverse modelling}

\subsection{Model and parameters}

We modelled all experiments with hygrothermal simulation software DELPHIN 6. The model considers two types of moisture transport: The vapour flux

$$
j_{v}=-K_{v}(\theta) \nabla p_{v}
$$

is driven by the vapour pressure gradient $\nabla p_{v}$, where $\mathrm{K}_{\mathrm{v}}(\theta)$ is the moisture-dependent vapour conductivity. The capillary liquid water flux

$$
j_{l}=-K_{l}(\theta)\left(\nabla p_{c}+\rho g\right)
$$

is driven by the capillary pressure gradient $\nabla p_{c}$, where $\mathrm{K}_{l}(\theta)$ is the liquid water conductivity dependent on moisture content. The volumetric moisture content is a function of the capillary pressure

$$
\theta=\theta\left(p_{c}\right)
$$

which is called the moisture storage function. Moreover, heat transport is considered and the thermal conductivity $\lambda$ is a function of moisture content

$$
\lambda=\lambda(\theta)
$$

This applies to the thermal capacity as well, however, its dependency from the moisture content can be considered as linear additive. Since the moisture storage function (3) is measured directly using desiccators and pressure plates, only $\mathrm{K}_{\mathrm{v}}(\theta), \mathrm{K}_{\mathrm{l}}(\theta)$ and $\lambda(\theta)$ need to be determined. Therefore, we used two different modelling approaches:

Continuous functions: We model $\mathrm{K}_{\mathrm{v}}(\theta)$ according to the serial-parallel approach and for $\mathrm{K}_{1}(\theta)$ we use the bundle of tubes model, both described in [2]. $K_{l}(\theta)$ is therefore derived from the moisture storage function applying the Hagen-Poiseuille equation. For the moisture storage function, the Gauss cumulative approach

$$
\theta(p C)=\sum_{i=1}^{N} \frac{\Delta \theta_{i}}{\sqrt{2}}\left(1+\operatorname{erf}\left(\frac{p C_{i}-p C}{\sqrt{2} S_{i}}\right)\right)
$$

is used according to [2]. The resulting liquid water conductivity according to the bundle of tubes model reads

$$
K_{l}(\theta)=\int_{0}^{\theta_{e f f}} p c(\theta)^{-2} d \theta
$$

Here the capillary pressure is $p C=\log \left(-p_{c}\right)$ and $\theta_{\text {eff }}$ is the moisture content at free saturation. This leads to three parameters for each modality in equation (5), which are $\Delta \theta_{i}, \mathrm{pC}_{\mathrm{i}}$ and $S_{i}$. We use two modalities $\mathrm{N}=2$ so that we obtain a total of 6 parameters. However, there is the constraint $\Delta \theta_{1}+\Delta \theta_{2}=\theta_{\text {eff }}$ so that eventually only 5 parameters are available to determine $\mathrm{Kl}(\theta)$.

Linear splines: We model $\mathrm{K}_{\mathrm{v}}$ and $\mathrm{K}_{1}$ using linear splines with $\mathrm{N}_{\mathrm{v}}$ and $\mathrm{N}_{1}$ points, respectively, which is similar as described in [4]. This approach dispenses a physical derivation of $\mathrm{K}_{1}$, however it allows to freely determine both functions.

In both approaches, the thermal conductivity is assumed a linear function where the value at dry conditions can be measured directly, so that there is only one value left to determine. In total this leads to $\mathrm{N}_{\mathrm{par}}=\mathrm{N}_{\mathrm{v}}+\mathrm{N}_{\mathrm{l}}+1$ parameters that need to be identified.

\subsection{Parameter identification algorithm}

Common experiments for the identification of the described parameters are the vapour cup test, where the vapour flux through a material under defined boundary conditions is measured [5] and the water uptake test, where the absorption of a material in direct contact with liquid water is measured [6]. Moreover, the drying experiment was introduced [7], where a saturated material is subjected to a constant ambient climate and the mass loss is measured.

For the parameter identification with inverse modelling, we consider these experiments together with the capillary condensation redistribution experiment (CCR) as described in section 2. All experiments are set up as simulations in DELPHIN 6, applying boundary conditions from the measurements. The simulation procedure and the evaluation of results is implemented in the cost function of an optimization algorithm. We use a weighted normalized sum of squared errors to determine the deviation between each measurement $y_{\text {meas }}$ and the corresponding simulation $\mathrm{y}_{\text {sim. }}$. First, we determine the total minimum $y_{\min }$ and total maximum $y_{\max }$ taking into account both simulation and measurement respectively. The normalization can then be described as

$$
f_{\text {norm }}(y)=\frac{y-y_{\min }}{\left(y_{\max }-y_{\min }\right)}
$$

so that the normalized sum of squared errors becomes

$$
\begin{gathered}
\text { sse }_{\text {norm }}= \\
\frac{w}{N} \sum_{i=1}^{N}\left(f_{\text {norm }}\left(y_{\text {meas }, i}\right)-f_{\text {norm }}\left(y_{\text {sim }, i}\right)\right)^{2}
\end{gathered}
$$

where w can be used to weight the respective experiment. The normalization holds the advantage that, with $\mathrm{w}=1$, each experiment is weighted equally in the cost function.

For optimization, we implemented different algorithms from [14] in a C++-based software, the Material Generator, which we expect to publish under non-commercial licence. The program allows optimizing material functions using different approaches (linear splines, continuous functions) taking into account multiple experiments, which can be determined by the user. Moreover, constraints are applied to the material functions $\mathrm{K}_{\mathrm{v}}$ and $\mathrm{K}_{\mathrm{l}}$, to ensure physically correct values. In particular, the number of local maxima in the $\mathrm{K}_{\mathrm{v}}$-spline is limited to $\leq 1$ and the $\mathrm{K}_{1}$-spline is constrained to $\mathrm{dK} / \mathrm{d} \theta>0$.

Eventually the Material Generator exports a material file (.m6) which provides the material functions for a DELPHIN simulation. 


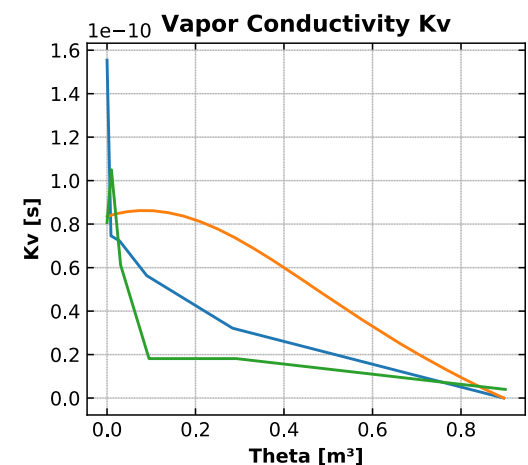

CCR average moisture content

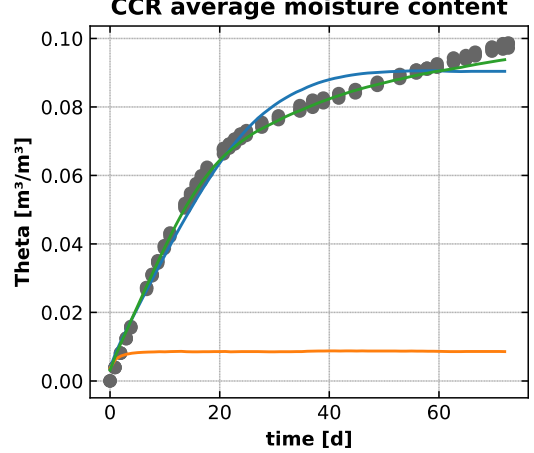

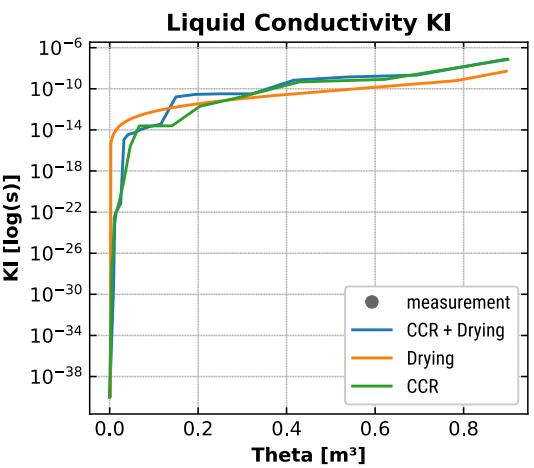
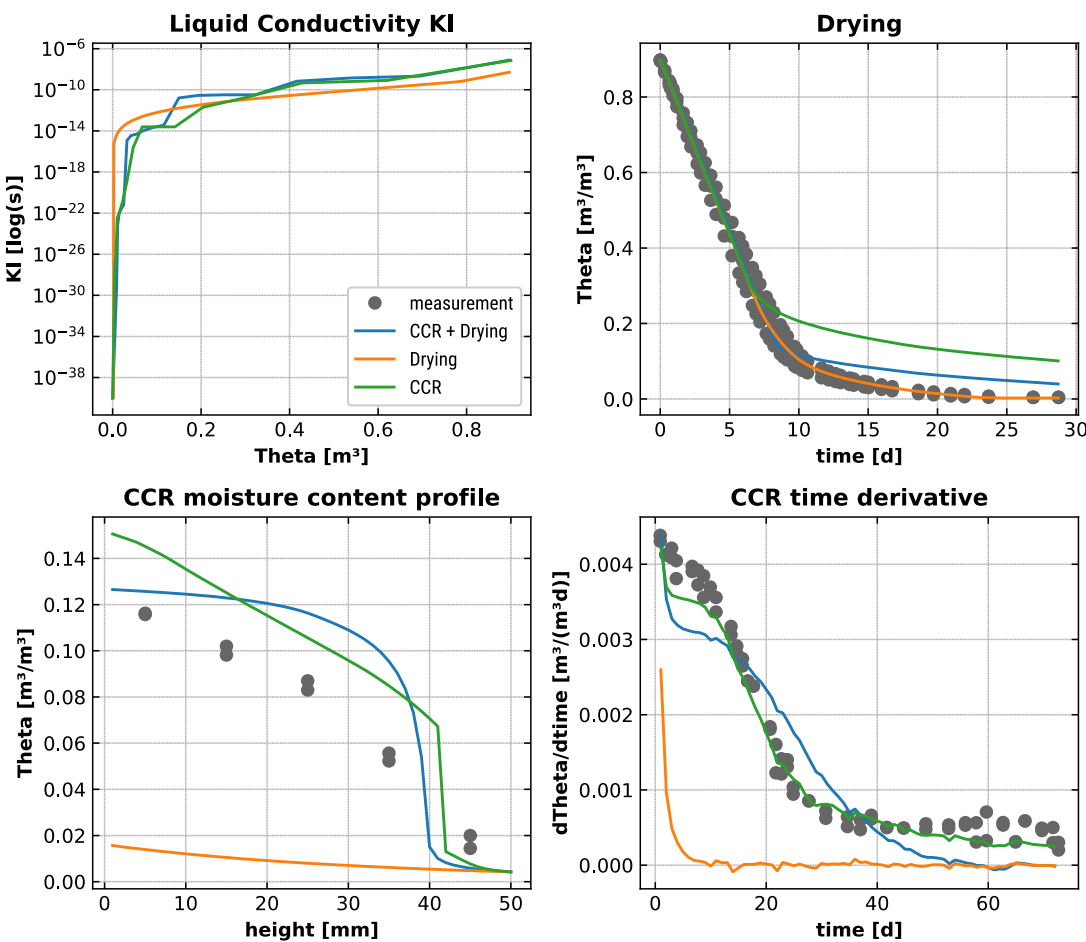

Fig. 8. CaSi-A material functions and measurements against inverse modelling results. For all parameter sets, vapour cup and water uptake experiments are taken into account. Additionally, the sets consider drying experiment (orange, continuous function), CCR experiment (green, linear spline) and both (blue, linear spline).
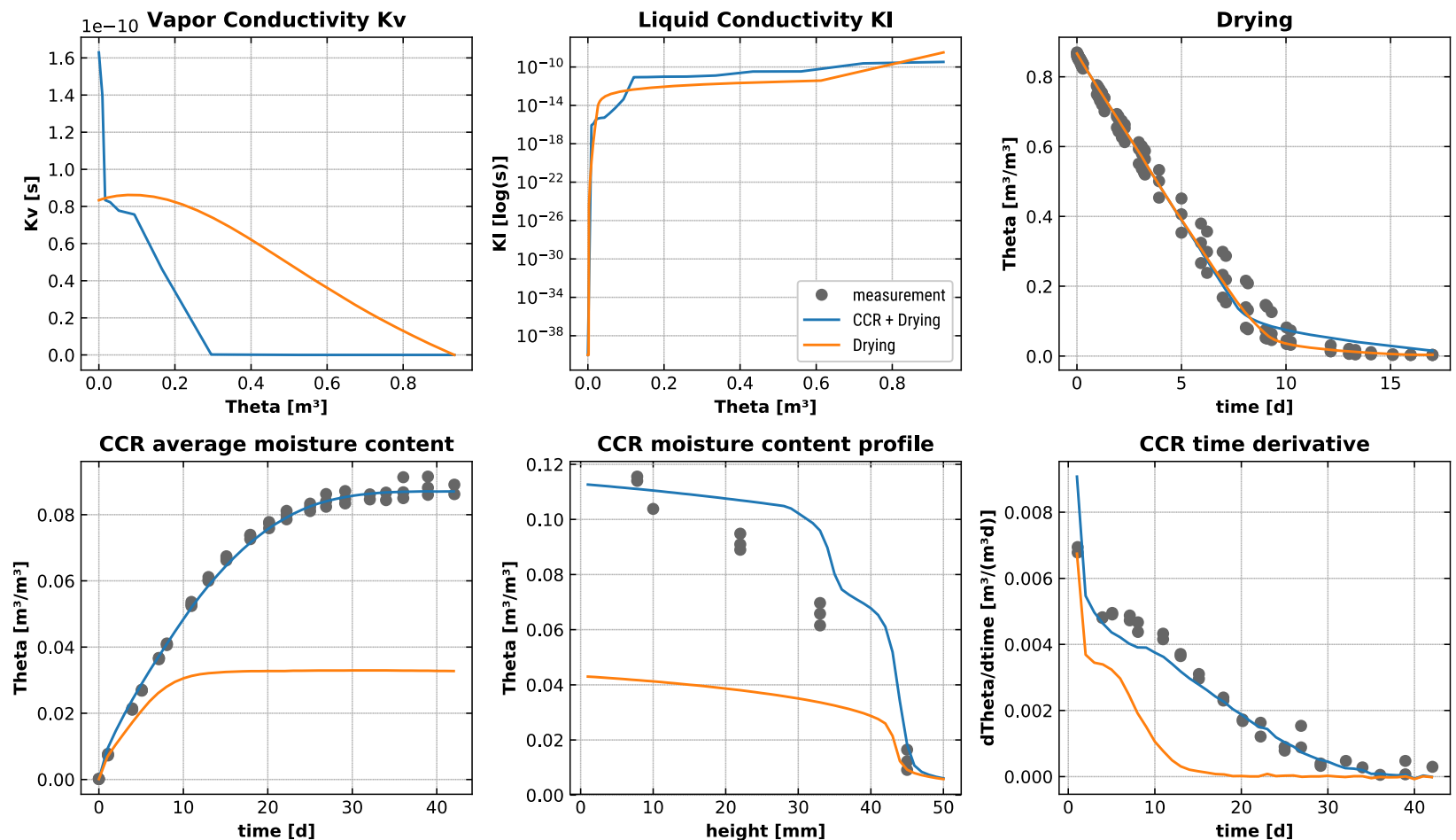

Fig. 9. CaSi-B material functions and measurements against inverse modelling results. For both parameter sets, vapour cup, water uptake and drying experiments are taken into account. The set represented by the blue line (continuous function) additionally considers the CCR experiment. 


\subsection{Results}

The material functions and simulation results of CaSi-A and $\mathrm{CaSi}-\mathrm{B}$ are shown in Figure 8 and Figure 9. For each parameter set, we conducted the inverse modelling taking into account vapour cup, water uptake experiment and additionally drying and/or CCR.

For CaSi-A, we found, when only the drying experiment is considered (orange, continuous function), the model dramatically underestimates the absorbed moisture in the CCR experiment. It appears that, liquid transport is overestimated, since vapour cup and drying experiments provide not enough information in the mid-moisture range between 0.05 and $0.1 \mathrm{~m}^{3} / \mathrm{m}^{3}$. When considering it for inverse modelling, the CCR experiment can be matched accurately (green). The liquid conductivity is lower, so that more moisture stays inside the material. The moisture profile at the end of the CCR experiment still shows a slight deviation. However, this measurement involves more inaccuracies compared to the averaged moisture content, due to incorrect cutting of material slices or desorption of the material during the cutting and weighing process.

For CaSi-B, a better agreement of modelling and measurement is achieved, even when both CCR and drying are considered. However, for both materials there was no parameter set found that matches both absorption and desorption accurately. We assume this to be caused by the absorption-desorption-hysteresis, which the present model is not capable considering. The moisture storage function for both materials was determined measuring desorption only.

Given this limitation, the CCR experiment still leads to a drastic correction of the modelled absorption process and the resulting material functions are considered to predict real-world moisture contents in constructions significantly more accurate.

\section{Performance in case-study}

We use the case study initially presented in [3] to compare the obtained material parameter set against measurements of a test wall in a climate chamber exposed to changing climate conditions. The base construction consists of a wood particleboard, PE foil and cement fibreboard imitating the exterior wall with a lower thermal capacity. Attached is an interior insulation of $50 \mathrm{~mm}$ calcium silicate with system specific glue mortar and plaster as well as paint on the inner surface.

Figure 10 shows the boundary conditions and measured volumetric water content of the calcium-silicate insulation. The experiment runs for $\sim 170$ days and comprises periods of drying, condensation, drying and frostthaw-cycles. For modelling, the parameters of $\mathrm{CaSi}-\mathrm{A}$ and $\mathrm{CaSi}-\mathrm{B}$ are employed. It should be noted that none of both is identical to the calcium-silicate used in the case study.

The findings are similar to the ones shown above. Results achieved with CCR-calibrated material functions show very good agreement with measurements, having a slight deviation only during desorption. This holds true for both tested materials $\mathrm{CaSi}-\mathrm{A}$ and $\mathrm{CaSi}-\mathrm{B}$. The model also considers ice formation, so that even through the frost-thaw cycles measurements could be replicated rather well. On the other hand, the moisture content is dramatically underestimated when the CCR experiment is not taken into account. In particular when CaSi-A is used, the calculated moisture content is about 10 times lower than measured values.
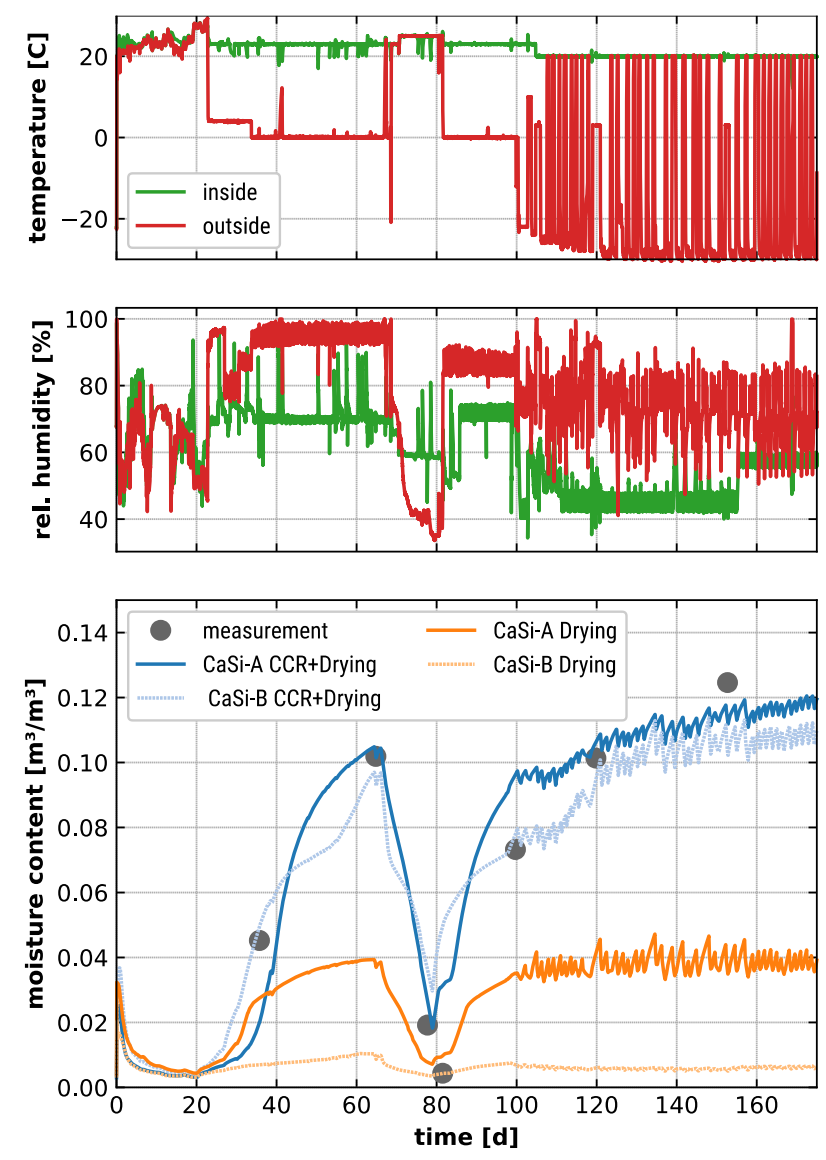

Fig. 10. Upper plots: temperature and relative humidity at interior and exterior wall. Lower plot: Measurements and simulations of the volumetric moisture content (average of an exterior $5 \mathrm{~mm}$ slice) applying the obtained material functions of $\mathrm{CaSi}-\mathrm{A}$ and $\mathrm{CaSi}-\mathrm{B}$.

\section{Conclusion}

We presented a non-isothermal experiment for material characterization, also known as capillary condensation redistribution test. We subjected three different materials, calcium-silicate (CaSi-A and $\mathrm{CaSi}-\mathrm{B}$ ) and autoclaved aerated concrete (AAC), to a warm and humid climate of $22{ }^{\circ} \mathrm{C}$ and $70 \%$ at the surface, while we kept the bottom at a temperature of $5{ }^{\circ} \mathrm{C}$. We measured the average moisture content of both materials in regular intervals over the entire period and eventually cut the samples into slices to measure their moisture profile.

$\mathrm{CaSi}$ gains considerably less moisture than AAC, which is partly caused by its higher thermal conductivity, resulting in a lower vapour pressure gradient. Moreover, we assume the higher liquid conductivity of CaSi to contribute.

In contrast to common experiments for material characterization, in the CCR experiment liquid and va- 
pour fluxes occur in opposite directions, which allows distinguishing between both. Therefore, we consider it crucial for the correct parameter identification. We presented an inverse modelling approach based on an optimization algorithm for parameter identification. Together with the CCR experiment, we model the drying, water uptake and vapour cup experiments in DELPHIN 6. The simulations are carried out and evaluated within the cost function of the optimization algorithm.

When model parameters are determined only with drying and vapour cup experiments, the simulation dramatically underestimates the moisture content in CCR measurements. Introducing CCR-calibration significantly enhances the model accuracy. All experiments can be reproduced sufficiently with a trade-off in the desorption behaviour. Eventually, we proved the model's fidelity on measurements of an interior insulation construction subjected to drying, condensation and freeze-thaw events over multiple months. The CCR-calibrated model could reliably reconstruct the moisture content, while the drying-calibrated model failed drastically.

\section{Acknowledgements}

The authors thankfully acknowledge the founding by the German Ministry of Economic Affairs and Energy (BMWi) under the project number 03ET1649A. Furthermore, we want to thank Frank Meissner for helpful discussions and technical support.

\section{References}

1. Innendämmung nach WTA II: Nachweis von Innendämmsystemen mittels numerischer Berechnungsverfahren (2014)

2. G. A. Scheffler, R. Plagge, International Journal of Heat and Mass Transfer, 53, 286-296 (2010)

3. P. Klõšeiko, K. Varda, T. Kalamees, Energy Procedia, 132, 525-530 (2017)

4. P. Klõšeiko, P. Freudenberg, MATEC Web Conf., 282, 02088 (2019)

5. ISO 12572 Hygrothermal performance of building materials and products - Determination of water vapour transmission properties - Cup method (2016)

6. R. Plagge, G. Scheffler, J. Grunewald, Bauphysik, 27, 315-323, (2005)

7. R. Plagge, G. Scheffler, Bauphysik, 27, 324-330, (2006)

8. A. Binder, D. Zirkelbach, H. M. Künzel, 10th Nordic Symposium on Building Physics, 2014

9. A. Binder, D. Zirkelbach, H. Künzel, International Conference on Porous Media and its Applications in Science, Engineering and Industry, Montecatini, (2010)

10. P. Häupl, H. Stopp, P. Strangfeld, H. Fechner, Bauphysik, 16, 138-147 (1994)

11. J. Grunewald, PhD Thesis, Dresden (1997)

12. A. Nicolai, PhD Thesis, Dresden (2007)
13. O. O. Adan, H. H. Brocken, J. E. Carmeliet, H. S. L. Hens, S. Roels und C.-E. Hagentoft, Introduction to the EC HAMSTAD Project, Journal of Building Physics, 27, 253-260 (2004)

14. S. G. Johnson, The NLopt nonlinear-optimization package, http://github.com/stevengj/nlopt, (2020) 